\title{
Unveiling Heterogeneity of Interfacial Water through the Water Bending Mode
}

\author{
Takakazu Seki, ${ }^{\dagger, \S}$ Shumei Sun, ${ }^{\dagger, \dagger, \S}$ Kai Zhong, ${ }^{\dagger}$ Chun-Chieh Yu, ${ }^{\dagger}$ Kevin Machel, ${ }^{\dagger}$ Lisa B. Dreier, ${ }^{\dagger}$
} Ellen H. G. Backus, ${ }^{\dagger, \ddagger}$ Mischa Bonn, ${ }^{\dagger}$ and Yuki Nagata* ${ }^{* \dagger}$

${ }^{\dagger}$ Max Planck Institute for Polymer Research, Ackermannweg 10, 55128 Mainz, Germany

${ }^{\ddagger}$ Department of Physical Chemistry, University of Vienna, Währinger Strasse 42, 1090 Vienna, Austria

\section{Supporting Information}

ABSTRACT: The water bending mode provides a powerful probe of the microscopic structure of bulk aqueous systems because its frequency and spectral line shape are responsive to the intermolecular interactions. Furthermore, interpreting the bending mode response is straightforward, as the intramolecular vibrational coupling is absent. Nevertheless, bending mode has not been used for probing the interfacial water structure, as it has been yet argued that the signal is dominated by bulk effects. Here, through the sum-frequency generation measurement of the water bending mode at the water/air and water/charged lipid interfaces, we demonstrate that the bending mode signal is dominated not by the bulk but by the interface. Subsequently, we disentangle the hydrogen-bonding of water at the water/air interface using the bending mode frequency distribution and find distinct interfacial hydrogen-bonded structures, which can be directly related to the interfacial organization of water. The bending mode thus provides an excellent probe of aqueous interfacial structure.

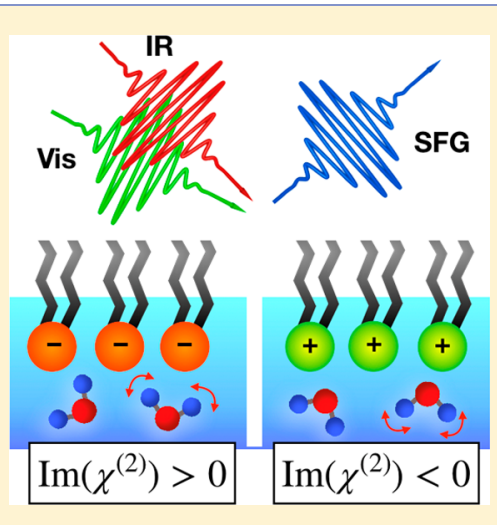

Tydrogen-bonds in the topmost water layer affect various 1 phenomena such as surface premelting of ice, ${ }^{1,2}$ the slipperiness of ice surfaces, ${ }^{3,4}$ evaporation of water ${ }^{5-7}$ or/and sublimation of ice, $^{8}$ the formation of aerosols, ${ }^{9}$ and the anomalously high surface tension of water. ${ }^{10}$ For example, a water molecule at the topmost layer at the water/air interface evaporates by breaking its hydrogen-bond with the interfacial water molecules. $^{5,6}$ It was suggested that a mobile water molecule rotates at the ice/air interface by breaking a hydrogen-bond with surrounding water molecules. ${ }^{3}$ As such, probing the structure of interfacial water to understand the details of the interfacial hydrogen-bonding is essential for uncovering the underlying physics of these phenomena.

Molecular-level insights into the structure of interfacial water have been obtained by using sum-frequency generation (SFG) spectroscopy. This technique can probe the vibrational modes of water, including the stretch, bending, and librational modes. ${ }^{11}$ The vast majority of SFG studies has focused on the $\mathrm{O}-\mathrm{H}$ stretch because of the strong signals and the high sensitivity of the $\mathrm{O}-\mathrm{H}$ stretch frequency to the hydrogen-bond strength of water. ${ }^{12}$ The $\mathrm{O}-\mathrm{H}$ stretch SFG spectra show a sharp $3700 \mathrm{~cm}^{-1}$ peak and a broad $3100-3500 \mathrm{~cm}^{-1}$ peak at both the water/air ${ }^{13}$ and ice/air interfaces. ${ }^{14}$ The $3700 \mathrm{~cm}^{-1}$ peak evidences the presence of dangling $\mathrm{O}-\mathrm{H}$ groups, while the $3100-3500 \mathrm{~cm}^{-1}$ band indicates a variety of the hydrogenbond interactions of interfacial water. ${ }^{15,16}$ However, the detailed distribution of hydrogen-bond strengths is not yet clear. Even with the time-resolved two-dimensional SFG technique, ${ }^{17,18}$ the heterogeneity of the hydrogen-bond at the water/air interface has been under debate; Bonn and co- workers pointed out a variation in the spectral diffusion time scales for various hydrogen-bonded $\mathrm{O}-\mathrm{H}$ stretch modes, ${ }^{19,20}$ whereas Tahara and co-workers showed insensitivity of the spectral diffusion to the stretch frequency. ${ }^{21,22}$ Difficulty in interpreting the $\mathrm{O}-\mathrm{H}$ stretch mode data arises from the fact that the $\mathrm{O}-\mathrm{H}$ stretch spectra are critically affected by the stretch-bend Fermi resonance $23-26$ and intermolecular coupling of the stretch chromophores. ${ }^{26-28}$ As such, the strengths of the individual hydrogen-bonds have not been fully clarified yet.

The $\mathrm{H}-\mathrm{O}-\mathrm{H}$ bending mode can be used as an excellent probe for resolving the individual hydrogen-bond strengths for the following two reasons. First, the bending mode frequency is highly correlated with $\mathrm{O}-\mathrm{H}$ stretch mode frequency, ${ }^{29-31}$ thus being a reporter for the hydrogen-bond strengths of water. ${ }^{12}$ Second, unlike the $\mathrm{O}-\mathrm{H}$ stretch mode, the intramolecular coupling of the bending modes is absent. ${ }^{28,30-32}$ Despite these advantages, the bending mode of interfacial water molecules has not been systematically investigated because its origin in the SFG spectra remains controversial. $^{31,33,34}$ Simulations predict a positive-negative SFG feature at the water/air interface, by assuming that the vibrational features reflect the contribution of the transition dipole moment at interfaces (interfacial dipole contribution). ${ }^{30,32,35}$ This assignment has been used for the fit of the experimental intensity spectra. ${ }^{33,36}$ In contrast, the heterodyne-detected SFG

Received: September 17, 2019

Accepted: October 24, 2019

Published: October 24, 2019 
line shape differed from that predicted by simulations, which was attributed to a higher-order quadrupole contribution from the bulk. ${ }^{34}$ If a signal is indeed masked by a bulk quadrupole contribution, information on the interfacial water molecules cannot be accessed via the bending mode measurements. Hence, elucidating the origin of the bending mode feature is essential for extracting from the SFG spectra the information on the interfacial water structure.

Here, by probing the $\mathrm{H}-\mathrm{O}-\mathrm{H}$ bending mode at the water/ charged lipid and water/air interfaces, we reveal that an SFG bending mode signal arises not from the bulk but from the interfacial dipole contribution. This opens up a new possibility of the $\mathrm{H}-\mathrm{O}-\mathrm{H}$ bending mode to probe hydrogen-bond network of interfacial water and ice. Subsequently, we demonstrate how the heterogeneity of the hydrogen-bond strength of interfacial water can be probed with the $\mathrm{H}-\mathrm{O}-\mathrm{H}$ bending mode.

First, we examine whether the bending mode signal originates from the interface or the bulk. When the transition dipole moment of the interfacial water molecules is small, an SFG signal may arise from the higher-order contributions such as the bulk quadrupole moment. ${ }^{37}$ A previous study has attributed the origin of the SFG signal to the bulk quadrupole contribution. ${ }^{34} \mathrm{~A}$ key to identify the interfacial dipole contribution vs the bulk quadrupole contribution is to examine whether the bending mode signal of the interfacial water $\left(\chi_{\text {bend }}^{(2)}\right)$ changes its sign upon the change of the orientation of the water molecules. If $\chi_{\text {bend }}^{(2)}$ originates from the interfacial dipole contribution, flipping the orientation of water molecules leads to a sign change of $\operatorname{Im}\left(\chi_{\text {bend }}^{(2)}\right)$. In contrast, if the bending mode is governed by the bulk quadrupole contribution, the sign of $\operatorname{Im}\left(\chi_{\text {bend }}^{(2)}\right)$ is unchanged. ${ }^{34}$ Thus, we identify the $\operatorname{Im}\left(\chi_{\text {bend }}^{(2)}\right)$ spectra at two, oppositely charged, interfaces where the water orientation is known to be flipped. ${ }^{38}$

Figure 1 plots the SFG intensity spectra at the water/ negatively charged lipid (1,2-dipalmitoyl-sn-glycero-3-phos-

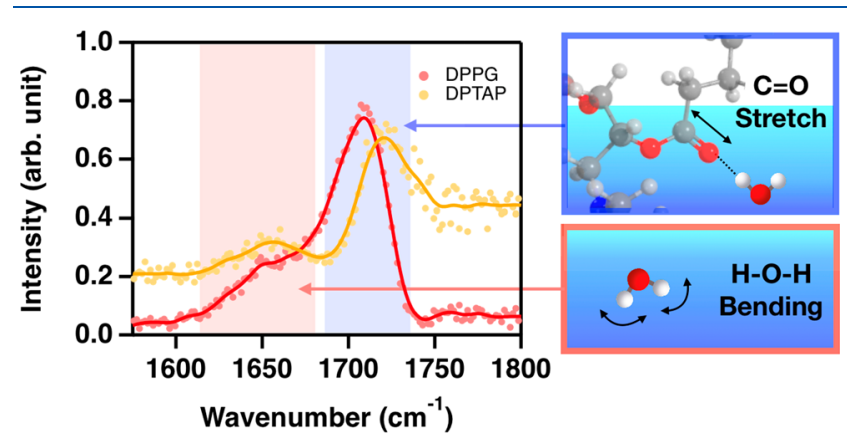

Figure 1. SFG spectra at the $\mathrm{H}_{2} \mathrm{O} / \mathrm{DPPG}$ and $\mathrm{H}_{2} \mathrm{O} / \mathrm{DPTAP}$ interfaces. The blue highlighted region contains the response from the $\mathrm{C}=\mathrm{O}$ stretch of the lipid headgroup. The red highlighted region contains the response from the water bending mode. Solid lines serve to guide the eyes.

pho-(1'-rac-glycerol), DPPG) and water/positively charged lipid (1,2-dipalmitoyl-3-trimethylammonium-propane, DPTAP) interfaces, respectively. Both spectra show the sharp $\mathrm{C}=\mathrm{O}$ stretch peak at $\sim 1710 \mathrm{~cm}^{-1}$ originating from the lipids, ${ }^{39}$ as well as the bending mode contribution around $\sim 1650 \mathrm{~cm}^{-1}$. The obtained bending mode contribution $\left(\chi_{\mathrm{eff}}^{(2)}\right)$, however, arises not only from $\left(\chi_{\text {bend }}^{(2)}\right)$ but also from the bulk dipole contribution $\left(\chi_{\text {bend }}^{(3)}\right)$. The $\chi_{\text {bend }}^{(2)}$ contribution can be disentangled from the $\chi_{\text {bend }}^{(3)}$ contribution by changing the ion concentration $c$ via ${ }^{40-43}$

$$
\chi_{\mathrm{eff}}^{(2)}(\omega)=\chi_{\text {bend }}^{(2)}(\omega)+\chi_{\text {bend }}^{(3)}(\omega) \Phi(c) \frac{\kappa(c)}{\kappa(c)-i \Delta k_{z}}
$$

where $\Phi, \kappa$, and $\Delta k_{z}$ denote the surface potential, the inverse of the Debye length, and the mismatch of the wave-vectors along the surface normal $(z$-axis) in the reflected SFG configuration, respectively.

Figure 2(a) displays the SFG spectra at the $\mathrm{H}_{2} \mathrm{O} / \mathrm{DPPG}$ and $\mathrm{D}_{2} \mathrm{O} / \mathrm{DPPG}$ interfaces with various ion concentrations. The difference between the $\mathrm{H}_{2} \mathrm{O}$ and $\mathrm{D}_{2} \mathrm{O}$ spectra can be found around $1650 \mathrm{~cm}^{-1}$, which signifies the $\mathrm{H}-\mathrm{O}-\mathrm{H}$ bending mode. The $\mathrm{D}-\mathrm{O}-\mathrm{D}$ bending mode frequency is at $\sim 1210$ $\mathrm{cm}^{-1}$, thus being absent in the measured frequency region. The difference between the $\mathrm{H}_{2} \mathrm{O}$ and $\mathrm{D}_{2} \mathrm{O}$ spectra is the most prominent at an ion concentration of $0.1 \mathrm{mM}$, while it disappears at $0.01-0.1 \mathrm{M}$ and reappears at $0.25-1 \mathrm{M}$. This trend is also apparent from the integrated spectral difference of $\mathrm{H}_{2} \mathrm{O}$ and $\mathrm{D}_{2} \mathrm{O}$ in Figure 2(b). The minimum around 0.01-0.1 $\mathrm{M}$ signifies the cancelation of the first and second terms of eq 1. This implies that the overall $\operatorname{Im}\left(\chi_{\mathrm{eff}}^{(2)}\right)$ changes its sign when the ion concentration is $0.01-0.1 \mathrm{M}$. For quantitative analysis, we performed spectral fitting for the SFG spectra by taking into consideration the nonresonant and $\mathrm{C}=\mathrm{O}$ stretch mode contributions as well as the $\chi_{\text {bend }}^{(2)}$ and $\chi_{\text {bend }}^{(3)}$ contributions. To identify the sign of $\operatorname{Im}\left(\chi_{\text {bend }}^{(2)}\right)$ uniquely from the intensity spectra, we used the information on the positive peak for the $\mathrm{C}=\mathrm{O}$ stretch mode in the imaginary spectra (see Supporting Information). ${ }^{39}$

The obtained $\operatorname{Im}\left(\chi_{\text {bend }}^{(2)}\right)$ and $\operatorname{Im}\left(\chi_{\text {bend }}^{(3)} \Phi\right)$ spectra, displayed in Figure 2(c), clearly illustrates that the amplitudes of these spectral features are comparable, and of opposite sign, explaining the signal cancellation around $0.1 \mathrm{M}$.

We then examined the SFG intensity spectra at the positively charged DPTAP interface. The $\mathrm{H}_{2} \mathrm{O}$ and $\mathrm{D}_{2} \mathrm{O}$ spectra are shown in Figure 3(a). Again, the $\mathrm{C}=\mathrm{O}$ stretch mode and bending mode contributions appear at $\sim 1710 \mathrm{~cm}^{-1}$ and $\sim 1650 \mathrm{~cm}^{-1}$, respectively. The $\operatorname{Im}\left(\chi_{\text {bend }}^{(2)}\right)$ and $\operatorname{Im}\left(\chi_{\text {bend }}^{(3)} \Phi\right)$ spectra obtained from the fit (see Supporting Information) are given in Figure 3(b), showing the negative $\operatorname{Im}\left(\chi_{\text {bend }}^{(2)}\right)$ and positive $\operatorname{Im}\left(\chi_{\text {bend }}^{(3)} \Phi\right)$. These are opposite to the signs at the water/DPPG interface, which are summarized in Figure 3(c).

An important consequence of the above analysis is the opposite signs of $\operatorname{Im}\left(\chi_{\text {bend }}^{(2)}\right)$ at the negatively charged DPPG and positively charged DPTAP interfaces. If the bulk quadrupole contribution would dominate the SFG signal, the signs of $\operatorname{Im}\left(\chi_{\text {bend }}^{(2)}\right)$ should be positive for both charged lipid interfaces, ${ }^{34}$ which contradicts with our observation. Therefore, the bending mode of water arises from the interfacial dipole contribution and thus can provide information on interfacial water. The signs of $\operatorname{Im}\left(\chi_{\text {bend }}^{(2)}\right)$ were further supported by density functional theory (DFT) calculation (see Supporting Information).

The SFG intensity spectrum at the water/air interface is displayed in Figure 4(a). The spectral line shape is similar to previously reported data. ${ }^{31-33}$ We fit the SFG intensity spectrum with two Lorentzians, which represent the bending modes of the water molecules with two hydrogen-bond donors (DD) and with one donor and the other free $\mathrm{O}-\mathrm{H}$ group (D) (see Supporting Information). ${ }^{30,32,35}$ The resonant frequencies obtained from the fit were 1661 and $1612 \mathrm{~cm}^{-1}$ for DD- and 

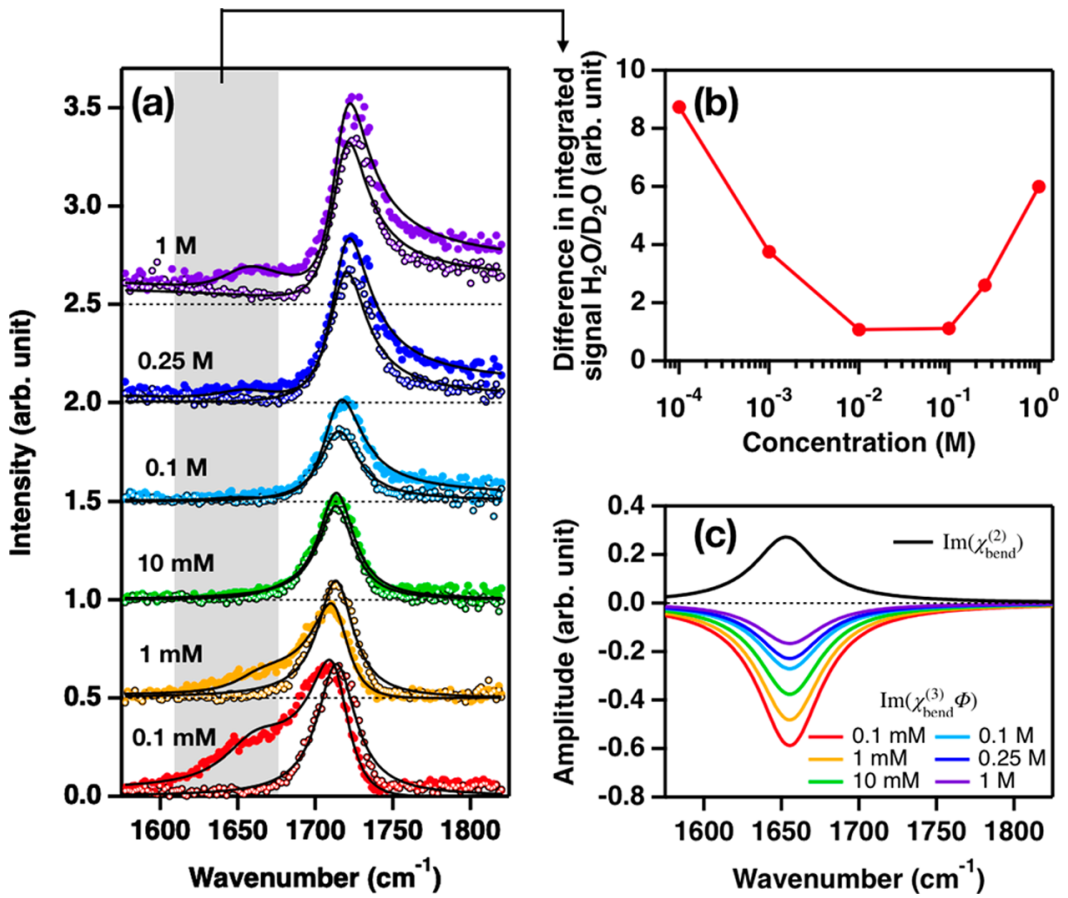

Figure 2. SFG spectra at the water/DPPG interface with various ion concentrations. (a) SFG spectra at the $\mathrm{H}_{2} \mathrm{O} / \mathrm{DPPG}$ (filled circles) and the $\mathrm{D}_{2} \mathrm{O} / \mathrm{DPPG}$ (open circles) interfaces. The black lines represent the fit. These spectra are offset by 0.5 for clarity. (b) Difference in the signal intensity between the $\mathrm{H}_{2} \mathrm{O}$ and $\mathrm{D}_{2} \mathrm{O}$ spectra integrated from 1610 to $1675 \mathrm{~cm}^{-1}$ (shaded region in (a)) vs ion concentration. (c) $\operatorname{Im}\left(\chi_{\text {bend }}^{(2)}\right)$ and $\operatorname{Im}\left(\chi_{\text {bend }}^{(3)} \Phi\right)$ spectra obtained from the fit.
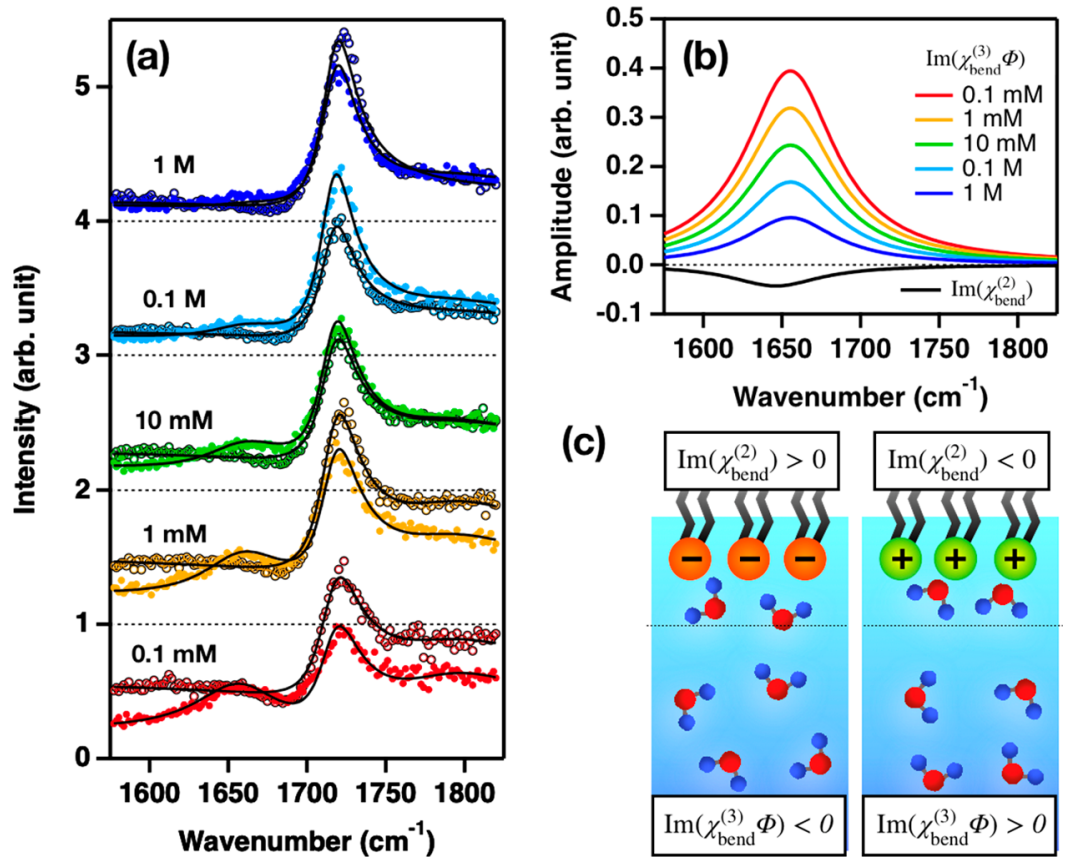

(c)

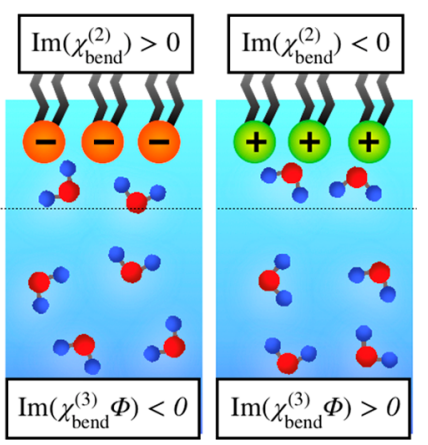

Figure 3. SFG spectra at the water/DPTAP interface with various ion concentrations. (a) SFG spectra at the $\mathrm{H}_{2} \mathrm{O} / \mathrm{DPTAP}$ (filled circles) and the $\mathrm{D}_{2} \mathrm{O} /$ DPTAP (open circles) interfaces. Black solid lines represent the fit. These spectra are offset with 1.0 for clarity. $(\mathrm{b}) \operatorname{Im}\left(\chi_{\text {bend }}^{(2)}\right)$ and $\operatorname{Im}\left(\chi_{\text {bend }}^{(3)} \Phi\right)$ spectra obtained from the fit. (c) Signs of $\operatorname{Im}\left(\chi_{\text {bend }}^{(2)}\right)$ and $\operatorname{Im}\left(\chi_{\text {bend }}^{(3)} \Phi\right)$ at the negatively and positively charged lipid interfaces.

D-type water molecules, respectively. This categorization is further supported by the sign of the peaks. The contribution from the D-type water molecules should have a negative sign, while the contribution from the DD-type water molecules should have a positive sign, ${ }^{32,35}$ which is consistent with our fit result.
Subsequently, we compared the $\operatorname{Im}\left(\chi_{\text {bend }}^{(2)}\right)$ spectra at the water/air, water/DPPG, and water/DPTAP interfaces in Figure 4(b): the amplitudes of $\operatorname{Im}\left(\chi_{\text {bend }}^{(2)}\right)$ at the water/air interface are comparable to or even larger than those of $\operatorname{Im}\left(\chi_{\text {bend }}^{(2)}\right)$ at the water/charged lipid interfaces. Thus, we conclude that $\chi_{\text {bend }}^{(2)}$ for the water/air interface arises not from the higher-order bulk quadrupole contribution but from the 


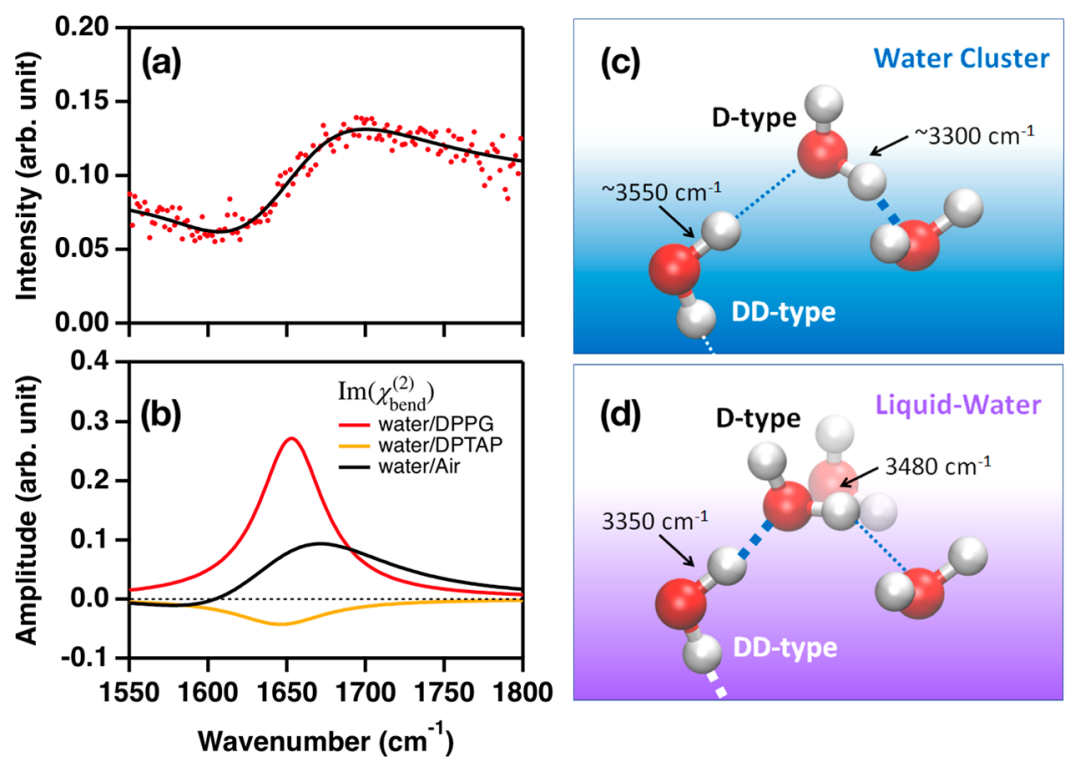

Figure 4. (a) SFG spectrum of the water/air interface. Black line represents the fit. (b) Comparison of $\operatorname{Im}\left(\chi_{\text {bend }}^{(2)}\right)$ spectra of the water/DPPG, water/DPTAP, and water/air interfaces. (c,d) Schematics of hydrogen-bonds of water at water/air interface for (c) water cluster at low temperature $^{44}$ and (d) liquid water/air interface. Strong (weak) hydrogen-bonds are represented by thick (thin) broken lines.

leading interfacial dipole contribution, in the same manner as for the water/charged lipid interface. The variation of the $\operatorname{Im}\left(\chi_{\text {bend }}^{(2)}\right)$ peak frequency further supports this conclusion because the bulk quadrupole frequency should be independent of surface species.

To gain further insight into the hydrogen-bonding of water at the water/air interface, we focus on the vibrational frequency. Table 1 summarizes the obtained bending mode

Table 1. Obtained Bending Mode Frequencies and Estimated Stretch Mode Frequencies ${ }^{a}$

\begin{tabular}{ccccc} 
& D-type & DD-type & $\begin{array}{c}\text { liquid } \\
\text { water }\end{array}$ & $\operatorname{Im}\left(\chi^{(3)}\right)$ \\
$\begin{array}{c}\mathrm{H}-\mathrm{O}-\mathrm{H} \text { bending } \\
\left(\mathrm{cm}^{-1}\right)\end{array}$ & $1612 \pm 12$ & $1661 \pm 10$ & $1650^{c}$ & $1655 \pm 1$ \\
$\begin{array}{c}\mathrm{O}-\mathrm{H} \text { stretch } \\
\left(\mathrm{cm}^{-1}\right)\end{array}$ & $3700)^{b}$ & $3353 \pm 39$ & $3400^{d}$ & $3381 \pm 4$ \\
\hline
\end{tabular}

${ }^{a}$ Calculation details can be found in the Supporting Information. ${ }^{b}$ Ref 16. ${ }^{c} \operatorname{Ref} 45 .{ }^{d} \operatorname{Ref} 11$.

and the corresponding stretch mode frequencies inferred from the bending mode frequencies, where a higher $\mathrm{H}-\mathrm{O}-\mathrm{H}$ bending frequency corresponds to a lower $\mathrm{O}-\mathrm{H}$ stretch frequency, i.e., stronger hydrogen-bonding. ${ }^{30,46}$ The frequencies of $3480 \pm 47 \mathrm{~cm}^{-1}$ for the hydrogen-bonded $\mathrm{O}-\mathrm{H}$ stretch of a D-type water molecule agrees with the previous study reporting $3510 \mathrm{~cm}^{-1}$. $^{47}$ A DD-type water molecule is estimated to have an $\mathrm{O}-\mathrm{H}$ stretch mode at $3353 \pm 39 \mathrm{~cm}^{-1}$, which is lower than both the frequency of $3480 \mathrm{~cm}^{-1}$ for the hydrogenbonded $\mathrm{O}-\mathrm{H}$ group of the $\mathrm{D}$-type molecule ${ }^{47}$ and that of 3400 $\mathrm{cm}^{-1}$ for bulk liquid water. This assignment is consistent with the recent theoretical analysis of the water-air interface. ${ }^{48}$ Clearly, the DD-type molecules at the water/air interface have stronger hydrogen-bond donors than the D-type water molecule and bulk water. This demonstrates that the hydrogen-bonds of the interfacial water are heterogeneous. Furthermore, these two types of hydrogen-bonded $\mathrm{O}-\mathrm{H}$ groups account for the different vibrational dynamics of the interfacial water; ${ }^{19}$ the slower (faster) spectral diffusion of $\mathrm{O}-$
$\mathrm{H}$ around $3500 \mathrm{~cm}^{-1}\left(3100-3400 \mathrm{~cm}^{-1}\right)$ can be attributed to the hydrogen-bonded $\mathrm{O}-\mathrm{H}$ group of the $\mathrm{D}$-type (DD-type) water molecules.

The lower frequency of the hydrogen-bonded $\mathrm{O}-\mathrm{H}$ group for the DD-type molecules $\left(3353 \mathrm{~cm}^{-1}\right)$ than that for the Dtype molecule $\left(3480 \mathrm{~cm}^{-1}\right)$ at the water/air interface is in contrast with the situation of water cluster surface, a model of the ice surface. For water clusters, the DD-type molecules have higher frequency $\mathrm{O}-\mathrm{H}$ group $\left(3550 \mathrm{~cm}^{-1}\right)$ than the D-type molecule $\left(\sim 3300 \mathrm{~cm}^{-1}\right){ }^{44}$ This means that the stretch frequency for the DD-type molecule is red-shifted with increasing temperature. This is surprising, as an increase in temperature usually blue-shifts the $\mathrm{O}-\mathrm{H}$ stretch mode. ${ }^{11}$ This leads to a picture that, with increasing temperature, the hydrogen-bonds of the DD-type molecules become strong (see Figure $4(\mathrm{c}, \mathrm{d}))$.

In summary, by measuring SFG spectra of the $\mathrm{H}-\mathrm{O}-\mathrm{H}$ bending mode of water at the water/charged lipid interfaces as well as the water/air interface, we examined the dipole contribution of the bending mode to the SFG signal. For charged lipid interfaces, we identified the $\chi_{\text {bend }}^{(2)}$ and $\chi_{\text {bend }}^{(3)}$ spectra and found the opposite signs of $\operatorname{Im}\left(\chi_{\text {bend }}^{(2)}\right)$ at the negatively charged DPPG and positively charged DPTAP interfaces. This suggests that the SFG signal of the bending mode arises from the interfacial dipole contribution. The comparable amplitudes of the $\operatorname{Im}\left(\chi_{\text {bend }}^{(2)}\right)$ peak at the water/air interface and water/charged lipid interfaces further demonstrate that the bending mode peak at the water/air interface is also dominated by the interfacial dipole contribution. Such a dipole contribution allows us to disentangle the heterogeneity of the interfacial water hydrogen-bond strengths. The bending mode frequency at the water/air interface indicates the stronger hydrogen-bond of a DD-type water than that of the $\mathrm{D}$-type water molecule with a free $\mathrm{O}-\mathrm{H}$ group. This trend is opposite between the water/air interface and water cluster surface, manifesting the drastic change of the heterogeneity of interfacial water with different phases.

Experimental Section. SFG Measurements. Our SFG measurements were performed on a femtosecond Ti:Sapphire 
amplified laser system (Coherent Libra, $\sim 800 \mathrm{~nm}, \sim 50 \mathrm{fs}, 1$ $\mathrm{kHz}$ ). The visible and IR beams overlapped spatially and temporally at the sample surface with their incident angles of $64^{\circ}$ and $40^{\circ}$ with respect to the surface normal, respectively. All the spectra were taken in $s s p$ (denoting $s$-, $s$-, and $p$ polarized SFG, visible, and IR, respectively) polarization combination and normalized to the nonresonant signal taken from the $z$-cut quartz. The details of the experiment can be found in the Supporting Information.

\section{ASSOCIATED CONTENT}

\section{S Supporting Information}

The Supporting Information is available free of charge on the ACS Publications website at DOI: 10.1021/acs.jpclett.9b02748.

Experimental procedures, fitting procedures, and DFT calculation (PDF)

\section{AUTHOR INFORMATION}

\section{Corresponding Author}

*E-mail: nagata@mpip-mainz.mpg.de.

ORCID

Ellen H. G. Backus: 0000-0002-6202-0280

Mischa Bonn: 0000-0001-6851-8453

Yuki Nagata: 0000-0001-9727-6641

\section{Author Contributions}

${ }^{\S}$ These authors contributed equally.

\section{Notes}

The authors declare no competing financial interest.

\section{ACKNOWLEDGMENTS}

We acknowledge financial support from MaxWater Initiative of the Max Planck Society and ERC Starting Grant (Grant No. 336679). We appreciate Prof. Doseok Kim for fruitful discussion.

\section{REFERENCES}

(1) Slater, B.; Michaelides, A. Surface Premelting of Water Ice. Nat. Rev. Chem. 2019, 3, 172-188.

(2) Nagata, Y.; Hama, T.; Backus, E. H. G.; Mezger, M.; Bonn, D.; Bonn, M.; Sazaki, G. The Surface of Ice under Equilibrium and Nonequilibrium Conditions. Acc. Chem. Res. 2019, 52, 1006-1015.

(3) Weber, B.; Nagata, Y.; Ketzetzi, S.; Tang, F.; Smit, W. J.; Bakker, H. J.; Backus, E. H. G.; Bonn, M.; Bonn, D. Molecular Insight into the Slipperiness of Ice. J. Phys. Chem. Lett. 2018, 9, 2838-2842.

(4) Louden, P. B.; Gezelter, J. D. Why Is Ice Slippery? Simulations of Shear Viscosity of the Quasi- Liquid Layer on Ice. J. Phys. Chem. Lett. 2018, 9, 3686-3691.

(5) Nagata, Y.; Usui, K.; Bonn, M. Molecular Mechanism of Water Evaporation. Phys. Rev. Lett. 2015, 115, 236102.

(6) Varilly, P.; Chandler, D. Water Evaporation: A Transition Path Sampling Study. J. Phys. Chem. B 2013, 117, 1419-1428.

(7) Persad, A. H.; Ward, C. A. Expressions for the Evaporation and Condensation Coefficients in the Hertz-Knudsen Relation. Chem. Rev. 2016, 116, 7727-7767.

(8) Haynes, D. R.; Tro, N. J.; George, S. M. Condensation and Evaporation of Water on Ice Surfaces. J. Phys. Chem. 1992, 96, 85028509.

(9) Reid, J. P.; Sayer, R. M. Heterogeneous Atmospheric Aerosol Chemistry: Laboratory Studies of Chemistry on Water Droplets. Chem. Soc. Rev. 2003, 32, 70-79.

(10) Israelachvili, J. N. Intermolecular and Surface Forces; Elsevier, 2011.
(11) Perakis, F.; De Marco, L.; Shalit, A.; Tang, F.; Kann, Z. R.; Kühne, T. D.; Torre, R.; Bonn, M.; Nagata, Y. Vibrational Spectroscopy and Dynamics of Water. Chem. Rev. 2016, 116, $7590-7607$.

(12) Rey, R.; Møller, K. B.; Hynes, J. T. Hydrogen Bond Dynamics in Water and Ultrafast Infrared Spectroscopy: A Theoretical Study. J. Phys. Chem. A 2002, 106, 11993-11996.

(13) Du, Q.; Superfine, R.; Freysz, E.; Shen, Y. R. Vibrational Spectroscopy of Water at the Vapor/Water Interface. Phys. Rev. Lett. 1993, 70, 2313-2316.

(14) Wei, X.; Miranda, P. B.; Shen, Y. R. Surface Vibrational Spectroscopic Study of Surface Melting of Ice. Phys. Rev. Lett. 2001, $86,1554-1557$.

(15) Nihonyanagi, S.; Kusaka, R.; Inoue, K.; Adhikari, A.; Yamaguchi, S.; Tahara, T. Accurate Determination of Complex $\chi(2)$ Spectrum of the Air/Water Interface. J. Chem. Phys. 2015, 143, 124707.

(16) Yamaguchi, S. Development of Single-Channel HeterodyneDetected Sum Frequency Generation Spectroscopy and Its Application to the Water/Vapor Interface. J. Chem. Phys. 2015, 143, 034202.

(17) Nihonyanagi, S.; Yamaguchi, S.; Tahara, T. Ultrafast Dynamics at Water Interfaces Studied by Vibrational Sum Frequency Generation Spectroscopy. Chem. Rev. 2017, 117, 10665-10693.

(18) Cyran, J. D.; Backus, E. H. G.; Nagata, Y.; Bonn, M. Structure from Dynamics : Vibrational Dynamics of Interfacial Water as a Probe of Aqueous Heterogeneity. J. Phys. Chem. B 2018, 122, 3667-3679.

(19) Hsieh, C.-S.; Okuno, M.; Hunger, J.; Backus, E. H. G.; Nagata, Y.; Bonn, M. Aqueous Heterogeneity at the Air/Water Interface Revealed by 2D-HD-SFG Spectroscopy. Angew. Chem., Int. Ed. 2014, 53, 8146-8149.

(20) Bonn, M.; Nagata, Y.; Backus, E. H. G. Molecular Structure and Dynamics of Water at the Water-Air Interface Studied with SurfaceSpecific Vibrational Spectroscopy. Angew. Chem., Int. Ed. 2015, 54, $5560-5576$

(21) Singh, P. C.; Nihonyahagi, S.; Yamaguchi, S.; Tahara, T. Communication: Ultrafast Vibrational Dynamics of Hydrogen Bond Network Terminated at the Air/Water Interface: A Two-Dimensional Heterodyne-Detected Vibrational Sum Frequency Generation Study. J. Chem. Phys. 2013, 139, 161101.

(22) Inoue, K.; Ishiyama, T.; Nihonyanagi, S.; Yamaguchi, S.; Morita, A.; Tahara, T. Efficient Spectral Diffusion at the Air/Water Interface Revealed by Femtosecond Time-Resolved HeterodyneDetected Vibrational Sum Frequency Generation Spectroscopy. J. Phys. Chem. Lett. 2016, 7, 1811-1815.

(23) Kananenka, A. A.; Skinner, J. L. Fermi Resonance in OHStretch Vibrational Spectroscopy of Liquid Water and the Water Hexamer. J. Chem. Phys. 2018, 148, 244107.

(24) Sovago, M.; Campen, R. K.; Wurpel, G. W. H.; Müller, M.; Bakker, H. J.; Bonn, M. Vibrational Response of Hydrogen-Bonded Interfacial Water Is Dominated by Intramolecular Coupling. Phys. Rev. Lett. 2008, 100, 173901.

(25) De Marco, L.; Ramasesha, K.; Tokmakoff, A. Experimental Evidence of Fermi Resonances in Isotopically Dilute Water from Ultrafast Broadband IR Spectroscopy. J. Phys. Chem. B 2013, 117, 15319-15327.

(26) Schaefer, J.; Backus, E. H. G.; Nagata, Y.; Bonn, M. Both Interand Intramolecular Coupling of $\mathrm{O}-\mathrm{H}$ Groups Determine the Vibrational Response of the Water/Air Interface. J. Phys. Chem. Lett. 2016, 7, 4591-4595.

(27) Pieniazek, P. A.; Tainter, C. J.; Skinner, J. L. Interpretation of the Water Surface Vibrational Sum-Frequency Spectrum. J. Chem. Phys. 2011, 135, 044701.

(28) Matt, S. M.; Ben-Amotz, D. Influence of Intermolecular Coupling on the Vibrational Spectrum of Water. J. Phys. Chem. B 2018, 122, 5375-5380.

(29) Falk, M. The Frequency of the H-O-H Bending Fundamental in Solids and Liquids. Spectrochim. Acta 1984, 40, 43-48. 
(30) Ni, Y.; Skinner, J. L. IR and SFG Vibrational Spectroscopy of the Water Bend in the Bulk Liquid and at the Liquid-Vapor Interface, Respectively. J. Chem. Phys. 2015, 143, 014502.

(31) Vinaykin, M.; Benderskii, A. V. Vibrational Sum-Frequency Spectrum of the Water Bend at the Air/Water Interface. J. Phys. Chem. Lett. 2012, 3, 3348-3352.

(32) Nagata, Y.; Hsieh, C.-S.; Hasegawa, T.; Voll, J.; Backus, E. H. G.; Bonn, M. Water Bending Mode at the Water-Vapor Interface Probed by Sum-Frequency Generation Spectroscopy: A Combined Molecular Dynamics Simulation and Experimental Study. J. Phys. Chem. Lett. 2013, 4, 1872-1877.

(33) Dutta, C.; Benderskii, A. V. On the Assignment of the Vibrational Spectrum of the Water Bend at the Air/Water Interface. J. Phys. Chem. Lett. 2017, 8, 801-804.

(34) Kundu, A.; Tanaka, S.; Ishiyama, T.; Ahmed, M.; Inoue, K.; Nihonyanagi, S.; Sawai, H.; Yamaguchi, S.; Morita, A.; Tahara, T. Bend Vibration of Surface Water Investigated by HeterodyneDetected Sum Frequency Generation and Theoretical Study: Dominant Role of Quadrupole. J. Phys. Chem. Lett. 2016, 7, 25972601.

(35) Moberg, D. R.; Straight, S. C.; Paesani, F. Temperature Dependence of the Air/Water Interface Revealed by Polarization Sensitive Sum-Frequency Generation Spectroscopy. J. Phys. Chem. B 2018, 122, 4356-4365.

(36) Dutta, C.; Mammetkuliyev, M.; Benderskii, A. V. ReOrientation of Water Molecules in Response to Surface Charge at Surfactant Interfaces. J. Chem. Phys. 2019, 151, 034703.

(37) Shen, Y. R. Basic Theory of Surface Sum-Frequency Generation. J. Phys. Chem. C 2012, 116, 15505-15509.

(38) Nihonyanagi, S.; Yamaguchi, S.; Tahara, T. Direct Evidence for Orientational Flip-Flop of Water Molecules at Charged Interfaces: A Heterodyne-Detected Vibrational Sum Frequency Generation Study. J. Chem. Phys. 2009, 130, 204704.

(39) Dreier, L. B.; Bonn, M.; Backus, E. H. G. Hydration and Orientation of Carbonyl Groups in Oppositely Charged Lipid Monolayers on Water. J. Phys. Chem. B 2019, 123, 1085-1089.

(40) Wen, Y.-C.; Zha, S.; Liu, X.; Yang, S.; Guo, P.; Shi, G.; Fang, H.; Shen, Y. R.; Tian, C. Unveiling Microscopic Structures of Charged Water Interfaces by Surface-Specific Vibrational Spectroscopy. Phys. Rev. Lett. 2016, 116, 016101.

(41) Ohno, P. E.; Wang, H.; Geiger, F. M. Second-Order Spectral Lineshapes from Charged Interfaces. Nat. Commun. 2017, 8, 1032.

(42) Gonella, G.; Lütgebaucks, C.; De Beer, A. G. F.; Roke, S. Second Harmonic and Sum-Frequency Generation from Aqueous Interfaces Is Modulated by Interference. J. Phys. Chem. C 2016, 120, 9165-9173.

(43) Schaefer, J.; Gonella, G.; Bonn, M.; Backus, E. H. G. SurfaceSpecific Vibrational Spectroscopy of the Water/Silica Interface: Screening and Interference. Phys. Chem. Chem. Phys. 2017, 19, 16875-16880.

(44) Yang, N.; Duong, C. H.; Kelleher, P. J.; Mccoy, A. B.; Johnson, M. A. Deconstructing Water's Diffuse OH Stretching Vibrational Spectrum with Cold Clusters. Science 2019, 364, 275-278.

(45) Maréchal, Y. IR Spectroscopy of an Exceptional H-Bonded Liquid: Water. J. Mol. Struct. 1994, 322, 105-111.

(46) Falk, M. The Frequency of the H-O-H Bending Fundamental in Solids and Liquids. Spectrochim. Acta 1984, 40, 43-48.

(47) Stiopkin, I. V.; Weeraman, C.; Pieniazek, P. A.; Shalhout, F. Y.; Skinner, J. L.; Benderskii, A. V. Hydrogen Bonding at the Water Surface Revealed by Isotopic Dilution Spectroscopy. Nature 2011, 474, 192-195.

(48) Ni, Y.; Skinner, J. L. Communication: Vibrational SumFrequency Spectrum of the Air-Water Interface, Revisited. J. Chem. Phys. 2016, 145, 031103. 